\title{
THE IMBEDDING THEOREM FOR FIBRE BUNDLES $\left({ }^{1}\right)$
}

\author{
BY \\ SHIING-SHEN CHERN AND YI-FONE SUN
}

Introduction. In the theory of sphere bundles the imbedding theorem of Whitney-Steenrod $\left({ }^{2}\right)$ has played an important rôle, as it reduces the problem of the classification of sphere bundles to that of the homotopy classification of the mappings of the base space into a Grassmann manifold. With this theorem the characteristic ring (relative to a coefficient ring) of the sphere bundle can be defined as the image under the dual homomorphism of the cohomology ring of the Grassmann manifold. It is natural to ask whether an analogous theorem holds for any fibre bundle. The main purpose of this paper is to establish such a theorem, and to give some of its generalizations and extensions.

The paper is divided into five sections. $\$ 1$ gives the definitions of various notions concerning fibre bundles. The imbedding theorem and its proof, for the case that the base space is a finite polyhedron, are given in $\$ 2$. Its extension to the case of metric compact ANR (= absolute neighborhood retract) is given in $\S 3$. In $\$ 4$ we extend the notion of the product of two sphere bundles in the sense of Whitney $\left({ }^{3}\right)$ to general fibre bundles and prove a simultaneous imbedding theorem for the product of fibre bundles. A treatment is given in $\$ 5$ of the cases where the reference groups are the classical groups, namely, the orthogonal, the properly orthogonal, the general linear, the unitary, and the symplectic groups. As is well known, the former two cases give the sphere bundles.

1. Definitions and notations. The notion of a fibre bundle arises in a sense from problems which are concerned with the applications of topology, and is therefore somewhat complicated in abstract formulation. We give in this section the definition of various concepts connected with it. A novel feature consists in the definition of the topology of the fibre bundle in terms of the coordinate functions, which simplifies the treatment somewhat.

1. Fibre bundle. A fibre bundle, to be denoted by $\mathfrak{F}$ or $\left\{F, G ; X, B ; \psi, \phi_{U}\right\}$, consists of :

(1) A space $F$, called the director space, which is transformed by a topo-

Presented to the Society, November 26, 1949; received by the editors December 10, 1948.

(1) After the paper had been submitted for publication, Professor N. E. Steenrod informed us that the main result of this paper was also proved by him and will be included in his forthcoming monograph on fibre bundles. To him are also due some suggestions for improvements of our treatment.

(2) Whitney [5]; Steenrod [1]. Numbers in brackets refer to the bibliography at the end of the paper.

(3) Whitney [5], [6]. 
logical group of homeomorphisms $G$, called the reference group, such that the map $G \times F \rightarrow F$ defined by the operations of $G$ on $F$ is continuous.

(2) A set $X$, which will be given a natural topology in the course of definition;

(3) A space $B$, called the base space;

(4) A transformation $\psi$, called the projection, of $X$ onto $B$;

(5) A system of neighborhoods $\mathfrak{U}=\{U\}$ which cover $B$, such that to each $U$ there exists a one-one transformation

$$
\phi_{U}: U \times F \rightarrow \psi^{-1}(U)
$$

satisfying the condition $\psi \phi_{U}(b, y)=b, b \in U, y \in F$.

These entities are supposed to satisfy the following:

Paste Condition. For given $U, b$ with $b \in U$ denote by $\phi_{U, b}$ the one-one transformation of $F$ onto $\psi^{-1}(b)$ defined by $\phi_{U, b}(y)=\phi_{U}(b, y), y \in F$. If $b$ belongs to the neighborhoods $U$ and $V$, then $\phi_{V, b}^{-1} \phi_{U, b} \in G$ and depends continuously on $b \in U \cap V$.

We shall define a topology in $X$. Let $\{N\}$ be a base in $F$, and $W$ an open set contained in a neighborhood $U$ of $\mathfrak{u}$. $X$ is topologized by the condition that the sets $\phi_{U}(W \times N)$ form a base. With this topology $X$ is a space, $\psi$ is a mapping (that is, a continuous transformation), and $\phi_{U}$ are homeomorphisms. We shall call $X$ the total space, the homeomorphisms $\phi_{U}$ the coordinate functions, and the neighborhoods of $\mathfrak{U}$ the coordinate neighborhoods. For a given $b \in B$ the set $\psi^{-1}(b)$ is called the fibre at $b$. For simplicity we shall also say that $X$ is a fibre bundle over $B$.

Suppose $B^{\prime}$ be a subspace of $B$. As a covering of $B^{\prime}$ we take $\mathfrak{u}^{\prime}=\left\{U \cap B^{\prime} \mid U \in \mathfrak{U}\right\}$. Define

$$
X^{\prime}=\bigcup_{b \in B^{\prime}} \psi^{-1}(b) \subset X
$$

and the coordinate functions

$$
\phi_{U}^{\prime} \cap_{B^{\prime}}(b, y)=\phi_{U}(b, y), \quad b \in U \cap B^{\prime}, y \in F .
$$

Then $\mathfrak{F}^{\prime}=\left\{F, G ; X^{\prime}, B^{\prime} ; \psi, \phi_{U \cap B^{\prime}}^{\prime}\right\}$ is a fibre bundle with the base space $B^{\prime}$, which may be called the part of $\mathfrak{F}$ over $B^{\prime}$ and denoted by $\mathfrak{F} \mid B^{\prime}$.

Equally naturally we may define an extension of $\mathfrak{F}$. Let $I$ be the unit interval $0 \leqq t \leqq 1$. We consider the Cartesian product $B \times I$ and take $\{U \times I \mid U \in \mathfrak{U}\}$ to be its covering. Put

$$
X^{*}=\bigcup_{b \in B} \psi^{-1}(b) \times I
$$

and define

$$
\psi^{*}\left(\psi^{-1}(b) \times t\right)=b \times t, \quad \phi_{U \times I}^{*}(b \times t, y)=\phi_{U}(b, y) \times t .
$$


Then $\left\{F, G ; X^{*}, B \times I ; \psi^{*}, \phi_{U \times I}^{*}\right\}$ is a fibre bundle, to be denoted by $\mathfrak{F} \times I$. For a given value $t \in I$ we shall denote by $\mathfrak{F} \times t$ the contraction $\mathfrak{F} \times I \mid B \times t$. It is easy to verify that $\mathfrak{F} \times t, t \in I$, is equivalent to $\mathfrak{F} \times 0$.

The fibre bundle is called a sphere bundle if $F$ is a sphere and $G$ the group of orthogonal transformations of $F$. It is called a vector bundle if $F$ is a vector space and $G$ the group of linear transformations in $F$. The sphere bundle and the vector bundle are said to be oriented if $G$ is the group of proper orthogonal transformations and the group of linear transformations of positive determinant respectively.

2. Equivalence. Equivalence is here defined for two fibre bundles with the same $F, G, B$. Two fibre bundles $\left\{F, G ; X, B ; \psi, \phi_{U}\right\}$ and $\left\{F, G ; X^{*}, B ; \psi^{*}, \phi_{U^{*}}^{*}\right\}$ are called equivalent if there exists a homeomorphism $h$ of $X$ onto $X^{*}$, which satisfies the conditions:

(1) For each $b \in B, h\left(\psi^{-1}(b)\right)=\psi^{*-1}(b)$.

(2) To each $b \in B$ and any two neighborhoods $U, U^{*}$ containing $b$ of the coverings $\mathfrak{u}, \mathfrak{U}^{*}$, we have

$$
\phi_{U^{*}, b}^{*-1} h \phi_{U, b} \in G
$$

and depends continuously on $b \in U \cap U^{*}$.

Clearly this equivalence relation is reflexive, symmetric, and transitive. It therefore enables us to divide the fibre bundles with given $F, G, B$ into mutually disjoint equivalence classes. We shall use the notation $\equiv$ to denote equivalence.

3. Mapping and induced bundle. Given a fibre bundle $\mathfrak{F}=\{F, G ; X, B$; $\left.\psi, \phi_{U}\right\}$, and a mapping $f: A \rightarrow B$. We shall define a fibre bundle $\left\{F, G ; X^{*}, A\right.$; $\left.\psi^{*}, \phi_{U^{*}}^{*}\right\}$, called the induced bundle and to be denoted by $(\mathfrak{F} ; f: A)$ or $A(f)$, as follows: $X^{*}$ is the union $\bigcup_{a \in A} a \times \psi^{-1}(f(a))$, and

$$
\psi^{*}\left(a \times \psi^{-1}(f(a))\right)=a .
$$

The neighborhoods $U^{*}$ are defined to be the open sets $f^{-1}(U)$ so that $\left\{U^{*}\right\}$ is a covering of $A$. Then we define

$$
\phi_{U}^{*} \cdot(a, y)=a \times \phi_{U}(f(a), y) .
$$

It is easy to verify that the Paste Condition is satisfied.

4. Admissible mapping of fibre bundles. Let

$$
\mathfrak{F}=\left\{F, G ; X, B ; \psi, \phi_{U}\right\}, \quad F^{*}=\left\{F, G ; X^{*}, B^{*} ; \psi^{*}, \phi_{U}^{*}\right\}
$$

be two fibre bundles with the same $F, G$. We take the points $b \in B, b^{*} \in B^{*}$, and consider the fibres $\psi^{-1}(b), \psi^{*-1}\left(b^{*}\right) . A$ mapping $k: \psi^{-1}(b) \rightarrow \psi^{*-1}\left(b^{*}\right)$ is called admissible if $b, b^{*}$ have respectively the coordinate neighborhoods $U, U^{*}$, such that $\phi_{U^{*}, b^{*}}^{*-1} k \phi_{U, b} \in G$. Notice that the condition is independent of the choice of $U, U^{*}$. This definition also applies to the case that $\mathfrak{F}$ and $\mathfrak{F}^{*}$ are identical. 
A mapping

$$
h: X \rightarrow X^{*}
$$

is called admissible if the following conditions are satisfied:

(1) For each $b \in B, h\left(\psi^{-1}(b)\right)=\psi^{*-1}\left(b^{*}\right)$, where $b^{*} \in B^{*}$. It follows that $h$ induces a transformation $h^{\prime}: B \rightarrow B^{*}$ defined by $h^{\prime}(b)=b^{*}$. Because of our definition of the topologies in $X$ and $X^{*}$ the transformation $h^{\prime}$ is continuous.

(2) For each $b \in B$ the partial mapping $h \mid \psi^{-1}(b)$ is admissible. Moreover, if $U$ and $U^{*}$ are coordinate neighborhoods which contain $b$ and $b^{*}=h^{\prime}(b)$ respectively, the homeomorphism $\phi_{U^{*}, b^{*}}^{*-1} h \phi_{U, b} \in G$ depends continuously on $b \in U \cap h^{\prime-1}\left(U^{*}\right)$.

For simplicity $h$ is said to define an admissible mapping $h: \mathfrak{F} \rightarrow \mathfrak{F}^{*}$. Admissible mapping of fibre bundles generalizes the notion of equivalence.

The following theorem is easily verified:

THEOREM 1.1. If $h: \mathfrak{F} \rightarrow \mathfrak{F}^{*}$ is an admissible mapping, the induced bundle $\left(\mathfrak{F}^{*} ; h^{\prime}: B\right)$ is equivalent to $\mathfrak{F}$.

5. Principal fibre bundles; the operations $\tau$ and $\tau_{F}^{-1}$. Given a fibre bundle $\left\{F, G ; X, B ; \psi, \phi_{U}\right\}$, we shall, following Ehresmann $\left({ }^{4}\right)$, define its principal fibre bundle $\left\{G, G ; X^{*}, B ; \psi^{*}, \phi_{U}^{*}\right\}$, as follows:

Let $b \in U \subset B$. We denote by $G_{b}$ the set of functions of the form $\phi_{U, b} g$ for all $g \in G$. $G_{b}$ depends only on $b$; for, if $b \in V$, then $\phi_{V, b}=\phi_{U, b} g_{0}, g_{0} \in G$, and the set $\phi_{V, b} G$ is identical with $\phi_{U, b} G$. We put $X^{*}=\bigcup_{b \in B} G_{b}$ and define the projection $\psi^{*}$ to be $\psi^{*}\left(G_{b}\right)=b$. The director space is $G$, operated on by $G$ as the group of left translations.

The coordinate functions $\phi_{U}^{*}$ are defined by

$$
\phi_{U}^{*}(b, g)=\phi_{U, b} g
$$

If $b \in U, V$, and $g \in G$, we have $\phi_{V, b}=\phi_{U, b} g_{0}, g_{0} \in G$, and

$$
\phi_{U, b}^{*}(g)=\phi_{U, b} g, \quad \phi_{V, b}^{*}(g)=\phi_{V, b} g=\phi_{U, b} g_{0} g,
$$

so that $\phi_{V, b}^{*-1} \phi_{U, b}^{*}$ is the mapping $g \rightarrow g_{0}^{-1} g$ in $G$ and the Paste Condition is satisfied.

We shall write $\left\{G, G ; X^{*}, B ; \psi^{*}, \phi_{U}^{*}\right\}=\tau\left\{F, G ; X, B ; \psi, \phi_{U}\right\}$.

To the operation $\tau$ so defined there is an inverse operation. In fact, let $\mathfrak{F}^{*}=\left\{G, G ; X^{*}, B ; \psi^{*}, \phi_{U}^{*}\right\}$ be a principal fibre bundle, and let $F$ be a space operated on by the group $G$ such that the transformation $F \times G \rightarrow F$ defined by the group operation is continuous. Define

$$
X=\bigcup_{b \in B} \phi_{U, b}^{*} F, \quad \psi\left(\phi_{U, b}^{*} F\right)=b, \quad \phi_{U}(b, y)=\phi_{U, b}^{*} y, \quad y \in F .
$$

(4) Ehresmann [1]. 
Then $\left\{F, G ; X, B ; \psi, \phi_{U}\right\}$ is a fibre bundle, to be denoted by $\tau_{F}^{-1} \mathfrak{F}^{*}$. It follows from definition that the following relations hold:

$$
\tau_{F}^{-1} \tau \mathfrak{F} \equiv \mathfrak{F}, \quad \tau \tau_{F}^{-1} \mathfrak{F}^{*} \equiv \mathfrak{F}^{*} .
$$

Two fibre bundles $\mathfrak{F}_{1}$ and $\mathfrak{F}_{2}$ are called associated if $\tau \mathfrak{F}_{1} \equiv \tau \mathfrak{F}_{2}$. From the above relations we see that two associated bundles are equivalent if they have the same director space.

For later use we state here the following theorems, whose proofs are immediate:

TheOREM 1.2 (EhresmanN). Two fibre bundles are equivalent if and only if their principal bundles are equivalent.

It follows that the operations $\tau$ and $\tau_{F}^{-1}$ are defined for equivalence classes of fibre bundles.

THEOREM 1.3. Let $\mathfrak{F}$ be a fibre bundle and $A$ a space which is mapped by $f$ into the base space of $\mathfrak{F}$. Then

$$
(\tau \mathfrak{F} ; f: A) \equiv \tau(\mathfrak{F} ; f: A) .
$$

If $\mathfrak{F}$ is a principal fibre bundle, then

$$
\left(\tau_{F}^{-1} \mathfrak{F} ; f: A\right) \equiv \tau_{F}^{-1}(\mathfrak{F} ; f: A) .
$$

In other words, the induction of fibre bundles commutes with the operations $\tau$ and $\tau_{\bar{F}}^{-1}$.

In a principal fibre bundle it is possible to define a group of transformations, which will be of importance in several connections. In fact, let $\mathfrak{F}$ $=\left\{G, G ; X, B ; \psi, \phi_{U}\right\}$ be a principal bundle. Let $g_{0} \in G$. Then $g_{0}$ acts on $X$ as a right translation as follows:

$$
x \cdot g_{0}=\phi_{U, b}\left(\phi_{U, b}^{-1}(x) g_{0}\right),
$$

where $b=\psi(x)$ and $U$ is a neighborhood containing $b$. Clearly, the point in the right-hand side is independent of the choice of $U$. The mapping $x \cdot g_{0}$ induces a mapping on each fibre, and is in general not admissible. For any fixed $x_{0}$ the mapping of $G$ onto the fibre through $x_{0}$ given by $g \rightarrow x_{0} \cdot g$ is admissible.

6. Universal fibre bundle. Consider the fibre bundles with given $F, G, B$. A fibre bundle $\left\{F, G ; X, A ; \psi, \phi_{U}\right\}$ is called universal (relative to $F, G, B$ ) if the equivalence classes of fibre bundles over $B$ are in one-one correspondence with the homotopy classes of mappings $B \rightarrow A$, or, more precisely, if the following properties hold:

(1) Every bundle with the same $F, G, B$ is equivalent to the bundle induced by a mapping $B \rightarrow A$.

(2) The bundles induced by the mappings $f, g: B \rightarrow A$ are equivalent if and only if $f$ and $g$ are homotopic. 
It will be our main purpose to prove theorems on the existence of universal fibre bundles and to draw consequences therefrom.

7. Simple bundles. As an example we consider a class of fibre bundles called simple. There is a natural way to define from the product space $B \times F$ a bundle whose reference group $G_{0}$ consists of the identity. If $\mathfrak{F}$ is any bundle over $B$ with the director space $F$ and reference group $G$, then $G_{0} \subset G$, and $B \times F$ determines an equivalence class of bundles relative to $F, G, B$, of which $B \times F$ is a member. Each such bundle is said to be equivalent to the product bundle or simply a product bundle.

A fibre bundle $\mathfrak{F}=\left\{F, G ; X, B ; \psi, \phi_{U}\right\}$ is called parallelisable if it admits a cross section, that is, if there exists a mapping $\lambda: B \rightarrow X$ such that $\psi \lambda$ is the identity. $\mathfrak{F}$ is called simple if its principal bundle is parallelisable.

Suppose now that $\mathfrak{F}^{*}=\left\{G, G ; X^{*}, B ; \psi^{*}, \phi_{U}^{*}\right\}$ is a principal bundle over $B$ which is parallelisable with the cross section $\lambda: B \rightarrow X^{*}$. Define $f: B \times G \rightarrow X^{*}$ by $f(b, g)=\lambda(b) \cdot g$. Then $f$ provides an equivalence of $B \times G$ and $\mathfrak{F}^{*}$. Thus a principal bundle is a product bundle if and only if it is parallelisable.

Now let $\mathfrak{F}$ be a simple bundle. Then $\mathfrak{F}^{*}=\tau \mathfrak{F}$ is parallelisable and is hence a product bundle. It follows from Theorem 1.2 that $\mathfrak{F}$ is also a product bundle. Thus simple bundle means the same thing as product bundle.

If $\mathfrak{F}^{*}$ is a fibre bundle over $B^{*}$ and $f_{0}$ maps $B$ into a point of $B^{*}$, then the induced bundle is a product bundle. Hence, if $\mathfrak{F}^{*}$ is a universal bundle relative to $F, G, B$, the induced bundle $\left(\mathfrak{F}^{*} ; f: B\right)$ is a product bundle if and only if $f$ is homotopic to a constant.

2. The imbedding theorem for finite polyhedra. The imbedding theorem is concerned with sufficient conditions for the existence of universal fibre bundles. An answer will be given in this section for the case that the base space is a finite polyhedron. This generalizes the imbedding theorem of Whitney-Steenrod for sphere bundles.

1. Fibre bundles induced by homotopic mappings. Let $\mathfrak{F}$ be a fibre bundle with the base space $B, A$ a compact space, and $f_{0}, f_{1}: A \rightarrow B$ two mappings of $A$ into $B$. We shall, following a procedure due essentially to Steenrod( $\left.{ }^{5}\right)$, prove the theorem that $A\left(f_{0}\right)$ and $A\left(f_{1}\right)$ are equivalent if $f_{0}$ and $f_{1}$ are homotopic. By the use of the notion of admissible mapping of fibre bundles the result can be put in a slightly more general form, which includes both the last mentioned result and the covering homotopy theorem as particular cases. This theorem can be stated as follows:

THEOREM 2.1. Let $\mathfrak{F}$ and $\mathfrak{F}^{\prime}$ be two fibre bundles with the base spaces $B$ and $B^{\prime}$ respectively, of which $B^{\prime}$ is compact. Let $f: \mathfrak{F}^{\prime} \times 0 \rightarrow \mathfrak{F}$ be an admissible mapping and $f^{\prime}: B^{\prime} \times I \rightarrow B$ an extension of its induced mapping $f_{0}^{\prime}: B^{\prime} \times 0 \rightarrow B$. Then there exists an admissible mapping $f: \mathfrak{F}^{\prime} \times I \rightarrow \mathfrak{F}$ which coincides with $f_{0}$ on $\mathfrak{F}^{\prime} \times 0$ and whose induced mapping is $f^{\prime}$.

(5) Steenrod [4, pp. 302-303]. 
Proof. Let $\mathfrak{F}=\left\{F, G ; X, B ; \psi, \phi_{U}\right\}, \mathfrak{F}^{\prime}=\left\{F, G ; X^{\prime}, B^{\prime} ; \psi^{\prime}, \phi_{U^{\prime}}^{\prime}\right\}$. For each $b \in B$ we select a pair of neighborhoods $V, W$ of $b$ such that $\bar{V} \subset W$ and that $\bar{W}$ is contained in some coordinate neighborhood $U$. Since $B^{\prime} \times I$ is compact, there exists a $\delta>0$ such that, for any point $b^{\prime} \in B^{\prime}$, the image $f^{\prime}\left(b^{\prime} \times I^{\prime}\right)$ is contained in some member of the family $\{V\}$, provided that $I^{\prime} \subset I$ is an interval of length less than $\delta$. Let $0=t_{0}<t_{1}<\cdots<t_{n}=1$ be a division of $I$, with $t_{k+1}-t_{k}<\delta$, and let $I_{k}$ denote the interval $t_{k} \leqq t \leqq t_{k+1}$.

Since, for each $b^{\prime}$, there exists a $V$ containing the image of $b^{\prime} \times I_{k}$ under $f^{\prime}$, it follows by continuity that there is a neighborhood $N$ of $b^{\prime}$ such that $f^{\prime}$ maps $N \times I_{k}$ into some $V$. Since $B^{\prime}$ is compact, we may select a finite covering by these neighborhoods: $N_{k, 1}, \cdots, N_{k, m}$. Let $V_{k, i} \supset f^{\prime}\left(N_{k, i} \times I_{k}\right)$ and let $W_{k, i}$ form a pair with $V_{k, i}$.

By the Urysohn lemma, there exists a continuous real-valued function $u_{k, i}(b)$ on $B$ such that $0 \leqq u_{k, i}(b) \leqq 1$ and

$$
u_{k, i}(b)=1, \quad b \in \bar{V}_{k, i}, \quad u_{k, i}(b)=0, \quad b \in B-W_{k, i} .
$$

Let

$$
\tau_{k, j}\left(b^{\prime}\right)=t_{k}+\left(t_{k+1}-t_{k}\right) \max _{i \leqq j}\left\{\min _{t_{k} \leqq t_{k+1}} u_{k, i}\left(f^{\prime}\left(b^{\prime}, t\right)\right)\right\} .
$$

Clearly $\tau_{k, j}$ is continuous, $t_{k} \leqq \tau_{k, j} \leqq t_{k+1}, \tau_{k, j}\left(b^{\prime}\right) \leqq \tau_{k, j+1}\left(b^{\prime}\right)$, and $\tau_{k, m}\left(b^{\prime}\right)=t_{k+1}$. We define by convention $\tau_{k, 0}\left(b^{\prime}\right)=t_{k}$.

The proof of the theorem hinges on the definition of a mapping

$$
f: \quad X^{\prime} \times I \rightarrow X
$$

with the desired properties. This will be achieved by double induction on $k$ and $j$. It is clearly sufficient to define

$$
f: \quad X^{\prime} \times I_{k} \rightarrow X
$$

for $\left(x^{\prime}, t\right) \in X^{\prime} \times I_{k}$ such that $\tau_{k, j}\left(b^{\prime}\right)<t \leqq \tau_{k, j+1}\left(b^{\prime}\right), \quad b^{\prime}=\psi^{\prime}\left(x^{\prime}\right)$, where $f\left(x^{\prime}, \tau_{k, j}\left(b^{\prime}\right)\right)$ is given.

Denote by $T$ the set of points $\left(b^{\prime}, t\right) \in B^{\prime} \times I$ such that $\tau_{k, j}\left(b^{\prime}\right)<t \leqq \tau_{k, j+1}\left(b^{\prime}\right)$. Then $\min _{t_{k} \leqq t_{\geqq} t_{k+1}} u_{k, j+1}\left(f^{\prime}\left(b^{\prime}, t\right)\right) \neq 0$, and we have $u_{k, j+1}\left(f^{\prime}\left(b^{\prime}, t\right)\right) \neq 0$ or $f^{\prime}\left(b^{\prime}, t\right) \in W_{k, j+1}$ for $\left(b^{\prime}, t\right) \in T$. It follows that $f^{\prime}(\bar{T}) \subset \bar{W}_{k, j+1}$, where the latter belongs to a coordinate neighborhood, say $U$, of $B$. Let $\phi$ be the coordinate function relative to $U$. Since $\phi$ establishes a homeomorphism between $U \times F$ and $\psi^{-1}(U)$, there exists a mapping $\zeta: \psi^{-1}(U) \rightarrow F$ such that $\zeta \phi(b, y)=y$ for $b \in U, y \in F$. Writing $b^{\prime}=\psi^{\prime}\left(x^{\prime}\right)$, we define

$$
f\left(x^{\prime}, t\right)=\phi\left(f^{\prime}\left(b^{\prime}\right), \zeta f\left(x^{\prime}, \tau_{k, j}\left(b^{\prime}\right)\right)\right) .
$$

This completes the induction. It is easy to verify that $f$ defines an admissible mapping $f: \mathfrak{F}^{\prime} \times I \rightarrow \mathfrak{F}$. 
REMARK. The theorem remains true if $B^{\prime}$ is the union of a countable number of compact spaces.

TheORem 2.2. Let $\mathfrak{F}$ be a fibre bundle with the base space $B, A$ a compact space, and $f_{0}, f_{1}: A \rightarrow B$ two mappings of $A$ into $B$. If $f_{0}$ and $f_{1}$ are homotopic, then $A\left(f_{0}\right)$ and $A\left(f_{1}\right)$ are equivalent.

Proof. It follows from Theorems 1.1 and 2.1 that

$$
\mathfrak{F} \times i \equiv A\left(f_{i}\right),
$$$$
i=0,1 \text {. }
$$

Since $\mathfrak{F} \times 0 \equiv \mathfrak{F} \times 1$, we get $A\left(f_{0}\right) \equiv A\left(f_{1}\right)$.

For the sake of completeness we state here the following useful theorem:

Theorem 2.3 (Covering homotopy THEOREM). If $X$ is the total space of $a$ fibre bundle over $B, A$ a compact space, $f$ a continuous map $A \rightarrow X$, and $h(a, t)$ a homotopy of the map $\psi f$ of $A$ into $B$, then there exists a homotopy $g(a, t)$ of $f$ which covers $h(a, t)$.

2. Equivalent fibre bundles. We shall first reduce the problem of universal fibre bundles to that of universal principal fibre bundles by means of the following theorem:

THEOREM 2.4. Let $G$ operate on $F$ such that the induced transformation $G \times F \rightarrow F$ is continuous. If $\mathfrak{F}_{0}$ is a universal principal fibre bundle relative to $G, G, B$, then $\tau_{F}^{-1} \mathfrak{F}_{0}$ is a universal fibre bundle relative to $F, G, B$.

Proof. Denote by $B_{0}$ the base space of $\mathfrak{F}_{0}$. Let $\mathfrak{F}=\left\{F, G ; X, B ; \psi, \phi_{U}\right\}$ be a fibre bundle. Then $\tau \mathfrak{F}$ is equivalent to the bundle $\left(\mathfrak{F}_{0} ; f: B\right)$ induced by a mapping $f: B \rightarrow A$. It follows that

$$
\mathfrak{F} \equiv \tau_{F}^{-1} \tau \mathfrak{F} \equiv \tau_{F}^{-1}\left(\mathfrak{F}_{0} ; f: B\right) \equiv\left(\tau_{F}^{-1} \mathfrak{F}_{0} ; f: B\right),
$$

by Theorem 1.3.

Consider next two induced bundles $\left(\tau_{\vec{p}}^{-1} \mathfrak{F}_{0} ; f: B\right)$ and $\left(\tau_{\vec{p}}^{-1} \mathfrak{F}_{0} ; g: B\right)$. They are equivalent if and only if $\tau_{F}^{-1}\left(\mathfrak{F}_{0} ; f: B\right)$ and $\tau_{F}^{-1}\left(\mathfrak{F}_{0} ; g: B\right)$ are equivalent, and hence if and only if $\left(\mathfrak{F}_{0} ; f: B\right)$ and $\left(\mathfrak{F}_{0} ; g: B\right)$ are equivalent. $\mathfrak{F}_{0}$ being a universal principal fibre bundle, a necessary and sufficient condition for the latter property is that $f$ and $g$ are homotopic.

TheOREM 2.5. Let $\mathfrak{F}=\left\{G, G ; X, B: \psi, \phi_{U}\right\}$ be a principal fibre bundle having as base space $B$ a polyhedron of dimension $n$. Let $\mathfrak{F}^{*}=\left\{G, G ; X^{*}, B^{*}\right.$; $\left.\psi^{*}, \phi_{U^{*}}^{*}\right\}$ be a principal fibre bundle such that $\pi_{i}\left(X^{*}\right)=0,0 \leqq i \leqq n-1$. Denote by $B_{0}$ a subpolyhedron of $B$ and by $\mathfrak{F}_{0}$ the part of $\mathfrak{F}$ over $B_{0}$. Then every admissible mapping $f_{0}: \mathfrak{F}_{0} \rightarrow \mathfrak{F}^{*}$ can be extended to an admissible mapping $f: \mathfrak{F} \rightarrow \mathfrak{F}^{*}$.

Proof. We take a simplicial decomposition of $B$ which is so fine that each simplex belongs to a coordinate neighborhood. By hypothesis there exists a 
mapping $f_{0}: \psi^{-1}\left(B_{0}\right) \rightarrow X^{*}$ such that $f_{0}\left(\psi^{-1}(b)\right)=\psi^{*-1}\left(b^{*}\right)$ for $b \in B_{0}$ and that the partial mapping $f_{0} \mid \psi^{-1}(b)$ is admissible. It is sufficient to define an extension $f$ of $f_{0}$ over $\psi^{-1}(B)$, with the desired properties. This extension $f$ will be defined on $\psi^{-1}\left(B_{0} \cup B^{r}\right)$, by induction on $r$, where $B^{r}$ denotes the $r$-dimensional skeleton of $B$.

For $r=0$ the definition of $f \mid \psi^{-1}\left(B_{0} \cup B^{0}\right)$ is obvious, it being only necessary to take $f \mid \psi^{-1}\left(\sigma^{0}\right)$ to be an admissible mapping into a fibre of $X^{*}$ for any 0 -dimensional simplex $\sigma^{0} \notin B_{0}$. Suppose $f \mid \psi^{-1}\left(B_{0} \cup B^{r-1}\right)$ be defined and let $\sigma^{r}$ be an $r$-simplex not belonging to $B_{0}$. Take a coordinate neighborhood $U$ containing $\sigma^{r}$, and denote by $e$ the identity of $G$. Then the map $f \phi_{U}\left(\partial \sigma^{r} \times e\right)$ is the map of an $(r-1)$-sphere into $X^{*}$ and is contractible. It follows that there is an extension $f \phi_{U}\left(\sigma^{r} \times e\right)$ of $f \phi_{U}\left(\partial \sigma^{r} \times e\right)$. Define then

$$
f \phi_{U}(b, g)=\left(f \phi_{U}(b, e)\right) \cdot g, \quad b \in \sigma^{r}, g \in G .
$$

Since $\phi_{U}: \sigma^{r} \times G \rightarrow \psi^{-1}\left(\sigma^{r}\right)$ is a homeomorphism, $f$ is defined for $\psi^{-1}\left(\sigma^{r}\right)$ and the induction is complete. It can be verified that $f$ defines an admissible mapping of $\mathfrak{F}$ into $\mathfrak{F}^{*}$.

From Theorem 2.5 we derive the following theorems which give sufficient conditions for a universal principal fibre bundle.

THeOREM 2.6. Let $\mathfrak{F}=\left\{G, G ; X, B ; \psi, \phi_{U}\right\}$ be a principal fibre bundle having as base space $B$ a polyhedron of dimension $n$. Let $\left\{G, G ; X^{*}, B^{*} ; \psi^{*}, \phi_{U^{*}}^{*}\right\}$ be a principal fibre bundle such that $\pi_{i}\left(X^{*}\right)=0,0 \leqq i \leqq n-1\left(^{6}\right)$. There exists a mapping $f: B \rightarrow B^{*}$ such that $\mathfrak{F} \equiv B(f)$.

Proof. Take $B_{0}$ to be empty and apply Theorem 2.5.

THEOREM 2.7. Let $\left\{G, G ; X^{*}, B^{*} ; \psi^{*}, \phi_{U^{*}}^{*}\right\}$ be a principal fibre bundle such that $\pi_{i}\left(X^{*}\right)=0,0 \leqq i \leqq n$. If $B$ is a polyhedron of dimension $n$ and $f, g: B \rightarrow B^{*}$ are mappings which induce equivalent fibre bundles $B(f) \equiv B(g)$, then the mappings $f$ and $g$ are homotopic.

Proof. Replacing, in Theorem 2.5, $\mathfrak{F}$ by $\mathfrak{F} \times I, B$ by $B \times I$, and $B_{0}$ by $(B \times 0) \cup(B \times 1)$, we get the theorem.

3. Existence of universal fibre bundles. From Theorems 2.2, 2.6, and 2.7, it is now easy to prove, by an explicit construction, the existence of universal fibre bundles for the case that the base space is a finite polyhedron and that the reference group is a linear group. The assumption on the reference group is reasonable in view of applications, as the most important case of compact Lie groups is included.

We denote by $A_{n}$ the general linear group in $n$ variables, and $A_{n}^{+}$the subgroup of all linear transformations of positive determinant of $A_{n}$. Let $I_{n}$ denote the identical linear transformation in $n$ variables. Then we can imbed $I_{m} \times A_{n} \subset A_{m+n}, I_{m} \times A_{n}^{+} \subset A_{m+n}^{+}$, by assuming that $I_{m}$ operates on the first $m$

( $\left.{ }^{(}\right) \pi_{0}\left(X^{*}\right)=0$ means by definition that $X^{*}$ is arcwise connected. 
and $A_{n}$ or $A_{n}^{+}$on the last $n$ variables. Using these notations, we have the theorem:

THEOREM 2.8. The homogeneous spaces

$$
A_{m+n} / I_{m} \times A_{n}, \quad A_{m+n}^{+} / I_{m} \times A_{n}^{+}, \quad m, n \geqq 1,
$$

are arcwise connected and have their homotopy groups $\pi_{i}=0$ for $1 \leqq i \leqq n-1$.

Proof. The two homogeneous spaces in question are homeomorphic. In fact, for $a \in A_{m+n}$, define the mapping

$$
a\left(I_{m} \times A_{n}\right) \rightarrow \eta a\left(I_{m} \times A_{n}^{+}\right), \quad \eta=I_{m+n-1} \times \operatorname{sgn} \operatorname{det} a .
$$

This induces a mapping

$$
A_{m+n} / I_{m} \times A_{n} \rightarrow A_{m+n}^{+} / I_{m} \times A_{n}^{+},
$$

which is easily proved to be a homeomorphism. It is therefore sufficient to consider the second space.

We have

$$
\pi_{i}\left(A_{m+n}^{+} / I_{m} \times A_{n}^{+}\right) \approx \pi_{i}\left(A_{m+n}^{+}, I_{m} \times A_{n}^{+}\right) .
$$

An element of the latter group is represented by the mapping of an $i$-cell into $A_{m+n}^{+}$with its boundary mapped into $I_{m} \times A_{n}^{+}$. By the covering homotopy theorem $\left({ }^{7}\right)$ we know that such a map is contractible into $I_{m} \times A_{n}^{+}$with its boundary fixed, if $i \leqq n-1$. The covering homotopy theorem also proves that the space $A_{m+n}^{+} / I_{m} \times A_{n}^{+}$is arcwise connected. Hence the theorem is proved.

Let $G$ be a linear group in $m$ variables. Then $A_{m+n+1} / I_{m} \times A_{n+1}$ is a fibre bundle over $A_{m+n+1} /\left(G \times A_{n+1}\right)$ with director space $G$ subject to the same group $G$ as left translations. By Theorems $2.2,2.6,2.7,2.8$, it follows that this fibre bundle is universal relative to $G, G, B$, if $B$ is a finite polyhedron of dimension $n$. Applying the operation $\tau_{F}^{-1}$ to this universal principal fibre bundle, we get a universal fibre bundle relative to $F, G, B$. This result is now stated in the following theorem:

Theorem 2.9. For fibre bundles whose base space $B$ is a finite polyhedron and whose reference group $G$ is a linear group, universal fibre bundles exist relative to $F, G, B$.

3. The imbedding theorem for compact metric ANR. We shall extend in this section the theorem on the existence of universal fibre bundles to cover the case that the base space is a compact metric ANR (absolute neighborhood retract). For this purpose it is convenient to make use of the notion of a bridge introduced by $\left.\mathrm{Hu}^{8}\right)$ in his study of mappings. We begin by recalling its definition and basic properties.

(7) Hurewicz-Steenrod [3, p. 64].

(8) $\mathrm{Hu}[2]$. 
1. Résumé of some theorems of $H u$. Let $X$ be a compact metric ANR, $X_{0}$ a closed subset of $X$, and $Y$ a connected ANR. We shall denote a finite open covering of $X$ by the notation $\alpha=\left\{a_{1}, \cdots, a_{r}\right\}$. Suppose the nerve of $\alpha$ be geometrically realized, and denote by $A$ the geometrical complex which realizes the nerve. The sets $X_{0} \cap a_{i}, i=1, \cdots, r$, form an open covering of $X_{0}$, and its realization $A_{0}$ is a subcomplex of $A$. Without danger of confusion we use the symbol $a_{i}$ to denote at the same time a set of the covering and a vertex of $A$. A mapping $\phi_{\alpha}: X \rightarrow A$ is called a canonical mapping of $\alpha$ if for each point $x \in X, \phi_{\alpha}(x)$ is contained in the closure of the simplex $a_{i_{0}} a_{i_{1}} \cdots a_{i_{m}}$ of $A$, where $a_{i_{0}}, \cdots, a_{i_{m}}$ are the members of $\alpha$ containing $x$.

Let $f: X_{0} \rightarrow Y$ be a given mapping and $\alpha$ a covering of $X$. A mapping $\psi_{\alpha}: A_{0} \rightarrow Y$ is called a bridge mapping for $f$ if the partial mapping $\psi_{\alpha} \phi_{\alpha} \mid X_{0}$ is homotopic to $f$ for each canonical mapping $\phi_{\alpha}: X \rightarrow A$ of the covering $\alpha$. If such a bridge mapping $\psi_{\alpha}$ exists, $\alpha$ is called a bridge for the mapping.

Concerning this notion of bridge $\mathrm{Hu}$ has established the following three basic theorems:

(1) Bridge REFINEMENT theOREM. For a given mapping $f: X_{0} \rightarrow Y$, any refinement $\beta$ of a bridge $\alpha$ is a bridge.

(2) BRIDGE EXISTENCE THEOREM. Every mapping $f: X_{0} \rightarrow$ Y has a bridge $\alpha$.

(3) BRIDGE HOMOTOPY THEOREM. If $\alpha, \beta$ are two bridges for a given mapping $f: X_{0} \rightarrow Y$, with the bridge mappings $\psi_{\alpha}: A \rightarrow Y, \psi_{\beta}: B \rightarrow Y$, there exists $a$ common refinement $\gamma$ of $\alpha$ and $\beta$ such that $\psi_{\alpha} p_{\gamma \alpha} \mid C_{0}$ and $\psi_{\beta} p_{\gamma \beta} \mid C_{0}$ are homotopic where $p_{\gamma \alpha}: C \rightarrow A, p_{\gamma \beta}: C \rightarrow B$ are arbitrary simplicial projections.

\section{The imbedding theorem.}

TheOREM 3.1. Let $B$ be a compact metric $A N R$ of dimension $n$, and let $F$ be the director space and $G$ the reference group. Let $\mathfrak{F}_{0}$ be a universal fibre bundle relative of $F, G, B^{\prime}$, where $B^{\prime}$ is a finite polyhedron of dimension $n$. Then $\mathfrak{F}_{0}$ is also a universal fibre bundle relative to $F, G, B$.

Proof. Consider the identity mapping $\iota: B \rightarrow B$. By the bridge existence theorem and the bridge refinement theorem there exist a bridge $\alpha$, a geometrical realization as an $n$-dimensional complex $A$ of the nerve of $\alpha$, and a bridge mapping $g: A \rightarrow B$ such that $g h$ is homotopic to $\iota$ for each canonical mapping $h: B \rightarrow A$. The mapping $g$ induces a bundle $A(g)$ over $A$. Since $A$ is an $n$-dimensional complex, there exists a mapping $s: A \rightarrow B_{0}$, where $B_{0}$ is the base space of $\mathfrak{F}_{0}$, such that $A(s) \equiv A(g)$. It follows that $B(s h) \equiv B(g h)$. Since $g h$ is homotopic to $\iota$, we have $B(g h) \equiv \mathfrak{F}$. Therefore $B(s h) \equiv \mathfrak{F}$, and the mapping $s h: B \rightarrow B_{0}$ induces a bundle over $B$, which is equivalent to the given bundle $\mathfrak{F}$.

Suppose next that $f_{0}, f_{1}: B \rightarrow B_{0}$ are mappings such that $B\left(f_{0}\right) \equiv B\left(f_{1}\right)$. We shall prove that then $f_{0} \simeq f_{1}$. In fact, introducing the bridge mapping $g$ and 
the canonical mapping $h$ as above, we have $A\left(f_{0} g\right) \equiv A\left(f_{1} g\right)$. Since $A$ is a finite complex, it follows from Theorem 2.7 that $f_{0} g \simeq f_{1} g$. Then $f_{0} g h \simeq f_{1} g h$. Since $g h \simeq_{l}$, we have $f_{i} g h \simeq f_{i}, i=0,1$. Consequently, we get $f_{0} \simeq f_{1}$, which is to be proved.

The above theorem reduces the question on the existence of universal fibre bundles for the case that the base space is a compact metric ANR to the case that the base space is a finite polyhedron.

3. The characteristic ring. The imbedding theorem permits us to define an important invariant of a fibre bundle, its characteristic ring. Let $\mathfrak{F}_{0}$, with the base space $B_{0}$, be a universal fibre bundle relative to $F, G, B$. Let $R$ be a commutative ring, and $H\left(B_{0}, R\right)$ the cohomology ring of $B_{0}$ with the coefficient ring $R$. The classes of equivalent fibre bundles with the same $F, G, B$ being in one-one correspondence with the homotopy classes of mappings $B \rightarrow B_{0}$, to each equivalence class of fibre bundles corresponds a definite ring homomorphism $H\left(B_{0}, R\right) \rightarrow H(B, R)$. We shall call the image of this ring homomorphism the characteristic ring relative to the coefficient ring $R$ and denote it by $C(B, R)$. The cohomology classes of $C(B, R)$ are called the characteristic cohomology classes.

The characteristic ring depends by definition on the choice of the universal fibre bundle $\mathfrak{F}_{0}$, which is by no means unique. It is very likely that the ring $C(B, R)$ as an abstract ring is independent of the choice of $\mathfrak{F}_{0}$, but we are not able to prove it $\left({ }^{9}\right)$.

4. Product of fibre bundles. In studying the problem of position of one sphere bundle in another we are naturally led to the notion of the product of sphere bundles. This section will be devoted to a discussion of the product of fibre bundles. A simultaneous imbedding theorem will be established, which is useful for the description of the position of one fibre bundle in another.

1. Definitions. Let

$$
\mathfrak{F}^{\prime}=\left\{F^{\prime}, G^{\prime} ; X^{\prime}, B ; \psi^{\prime}, \phi_{U}^{\prime}\right\}, \quad \mathfrak{F}^{\prime \prime}=\left\{F^{\prime \prime}, G^{\prime \prime} ; X^{\prime \prime}, B ; \psi^{\prime \prime}, \phi_{U}^{\prime \prime}\right\}
$$

be two fibre bundles, with the same base space $B$ and the same family of coordinate neighborhoods $\{U\}$. We shall define a fibre bundle, their product,

$$
\mathfrak{F}^{\prime} \times \mathfrak{F}^{\prime \prime}=\left\{F^{\prime} \times F^{\prime \prime}, G^{\prime} \times G^{\prime \prime} ; X, B ; \psi, \phi_{U}\right\}
$$

as follows: The director space is $F^{\prime} \times F^{\prime \prime}$ and is transformed by $G^{\prime} \times G^{\prime \prime}$ according to the formula $\left(g^{\prime} \times g^{\prime \prime}\right)\left(y^{\prime} \times y^{\prime \prime}\right)=g^{\prime}\left(y^{\prime}\right) \times g^{\prime \prime}\left(y^{\prime \prime}\right), g^{\prime} \in G^{\prime}, g^{\prime \prime} \in G^{\prime \prime}$, $y^{\prime} \in F^{\prime}, y^{\prime \prime} \in F^{\prime \prime}$. The total space is

$$
X=\bigcup_{b \in B} \phi_{U, b}^{\prime}\left(F^{\prime}\right) \times \phi_{U, b}^{\prime \prime}\left(F^{\prime \prime}\right)
$$

$\left.{ }^{9}\right)$ In the case of sphere bundles this can be proved by interpreting the generators of the characteristic ring as certain obstructions. 
while projection $\psi$ is defined by

$$
\psi\left\{\phi_{U, b}^{\prime}\left(F^{\prime}\right) \times \phi_{U, b}^{\prime \prime}\left(F^{\prime \prime}\right)\right\}=b .
$$

The coordinate functions are

$$
\phi_{U}\left(b, y^{\prime} \times y^{\prime \prime}\right)=\phi_{U, b}^{\prime}\left(y^{\prime}\right) \times \phi_{U, b}^{\prime \prime}\left(y^{\prime \prime}\right) .
$$

It is easily verified that the Paste Condition is satisfied.

This definition of the product bundle does not include the case of sphere bundles as defined by Whitney. We shall, however, show in the next section how, with the help of a relationship between sphere bundles and vector bundles, the product of vector bundles in the sense just defined will lead to the product of sphere bundles in the sense of Whitney.

2. Change of the reference group. In order to derive from our product of fibre bundles Whitney's product of sphere bundles, we shall study a relationship between fibre bundles with different reference groups. In fact, let $H$ be a subgroup of $G$. A fibre bundle with the reference group $H$ can be considered as a bundle with the group $G$. The converse question is solved by the following result of Ehresmann $\left({ }^{10}\right)$.

THEOREM 4.1. Let $\mathfrak{F}$ be a fibre bundle with the reference group $G$ and let $H$ be a subgroup of $G$. Construct from the principal bundle $\tau \mathfrak{F}$ the bundle $\tau_{G / H}^{-1} \tau \mathfrak{F}$ whose director space is the homogeneous space $G / H$ of left cosets of $G$ relative to $H$. $\mathfrak{F}$ is equivalent to a bundle with the reference group $H$ if and only if $\tau_{G / H}^{-1} \tau \mathfrak{F}$ is parallelisable.

Suppose the base space $B$ be given. Denote by $(B, G)$ an equivalence class of principal fibre bundles relative to $G, G, B$. If $H$ is a subgroup of $G$, then there is a natural correspondence $\lambda:(B, H) \rightarrow(B, G)$. From Theorem 4.1 it follows that the correspondence is onto if and only if the bundles of the class $\tau_{a / H}^{-1}(B, G)$ is parallelisable. If $B$ is a polyhedron of dimension $n$, a sufficient condition for this is $\pi_{i}(G / H)=0,0 \leqq i \leqq n-1$.

Suppose the bundles relative to $G, G, B$ have a universal bundle $\mathfrak{F}_{0}$, whose base space we denote by $B_{0}$. Two induced bundles $B\left(f_{0}\right)$ and $B\left(f_{1}\right)$ are equivalent if and only if $f_{0}$ and $f_{1}$ are homotopic, that is, if and only if there is a mapping $F: B \times I \rightarrow B_{0}$, with $F(B \times 0)=f_{0}, F(B \times 1)=f_{1}$. If we write $B^{\prime}=B$ $X I$, the mapping $F$ induces a bundle $B^{\prime}(F)$ over $B^{\prime}$, and the bundles $B\left(f_{0}\right)$ and $B\left(f_{1}\right)$ are equivalent relative to $H$ if $B^{\prime}(F)$ is equivalent relative to $G$ to a bundle with the reference group $H$. A necessary and sufficient condition for the latter property is that $\tau_{G / H}^{-1} B^{\prime}(F)$ is parallelisable. This condition is satisfied if $\pi_{i}(G / H)=0,0 \leqq i \leqq n$.

It follows, when $B$ is a polyhedron of dimension $n$, that the correspondence $\lambda$ is one-one and onto if $\pi_{i}(G / H)=0,0 \leqq i \leqq n$.

${ }^{(10)}$ Ehresmann [1]. 
When $G=A^{+}(n), H=0^{+}(n)$, the homogeneous space $G / H$ is a Euclidean space and is hence contractible. Therefore there is a one-one correspondence between classes of sphere bundles and classes of vector bundles.

3. The simultaneous imbedding theorem. It is important to remark that the above "product" admits an inverse operation. In fact, let $\left\{F^{\prime} \times F^{\prime \prime}, G^{\prime} \times G^{\prime \prime}\right.$; $\left.X, B ; \psi, \phi_{U}\right\}$ be a fibre bundle, where the reference group $G^{\prime} \times G^{\prime \prime}$ operates on the director space $F^{\prime} \times F^{\prime \prime}$ as in the last paragraph. We shall define two fibre bundles whose product is a fibre bundle equivalent to the given one. Write a point of $F^{\prime} \times F^{\prime \prime}$ in the form $y^{\prime} \times y^{\prime \prime}, y^{\prime} \in F^{\prime}, y^{\prime \prime} \in F^{\prime \prime}$, and define the projections $\zeta_{1}\left(y^{\prime} \times y^{\prime \prime}\right)=y^{\prime}, \zeta_{2}\left(y^{\prime} \times y^{\prime \prime}\right)=y^{\prime \prime}$. Let $b \in U \subset B$. Two points $z, z^{\prime}$ $\in \psi^{-1}(b)$ are called equivalent if $\zeta_{i}\left(\phi_{U, b}^{-1}(z)\right)=\zeta_{i}\left(\phi_{U, b}^{-1}\left(z^{\prime}\right)\right), i=1,2$. This equivalence relation is independent of the choice of the neighborhood $U$. The equivalence classes thus obtained form a decomposition space $Z_{i}(b), i=1,2$. Denote by $(z)_{i}$ the class which contains $z$. Put

$$
X^{\prime}=\bigcup_{b \in B} Z_{1}(b), \quad X^{\prime \prime}=\bigcup_{b \in B} Z_{2}(b),
$$

and define

$$
\begin{aligned}
\psi^{\prime}\left(Z_{1}(b)\right) & =b, \quad \psi^{\prime \prime}\left(Z_{2}(b)\right)=b, \\
\psi_{U}^{\prime}\left(b \times y^{\prime}\right) & =\left(\phi_{U}\left(b \times\left(y^{\prime} \times y^{\prime \prime}\right)\right)\right)_{1}, \\
\phi_{U}^{\prime \prime}\left(b \times y^{\prime \prime}\right) & =\left(\phi_{U}\left(b \times\left(y^{\prime} \times y^{\prime \prime}\right)\right)\right)_{2} .
\end{aligned}
$$

According to these definitions we get the fibre bundles

$$
\mathfrak{F}^{\prime}=\left\{F^{\prime}, G^{\prime} ; X^{\prime}, B ; \psi^{\prime}, \phi_{U}^{\prime}\right\}, \quad \mathfrak{F}^{\prime \prime}=\left\{F^{\prime \prime}, G^{\prime \prime} ; X^{\prime \prime}, B ; \psi^{\prime \prime}, \phi_{U}^{\prime \prime}\right\} .
$$

It is easily verified that $\mathfrak{F}^{\prime} \times \mathfrak{F}^{\prime \prime}$ is equivalent to the given fibre bundle.

Consider two fibre bundles

$$
\mathfrak{F}^{\prime}=\left\{F^{\prime}, G^{\prime} ; X^{\prime}, B ; \psi^{\prime}, \phi_{U}^{\prime}\right\}, \quad \mathfrak{F}^{\prime \prime}=\left\{{F^{\prime \prime}}^{\prime \prime} G^{\prime \prime} ; X^{\prime \prime}, B ; \psi^{\prime \prime}, \phi_{U}^{\prime \prime}\right\},
$$

with the same base space $B$ and their product bundle $\mathfrak{F}^{\prime} \times \mathfrak{F}^{\prime \prime}$. Such a triple of bundles we shall call a triad. We consider the triads with given $F^{\prime}, F^{\prime \prime}$, $G^{\prime}, G^{\prime \prime}, B$. A triad $\left\{\xi^{\prime}, \xi^{\prime \prime}, \xi^{\prime} \times \xi^{\prime \prime}\right\}$, with the director spaces $F^{\prime}, F^{\prime \prime}$, the reference groups $G^{\prime}, G^{\prime \prime}$, and the base space $A$, is called a universal triad of fibre bundles relative to $F^{\prime}, F^{\prime \prime}, G^{\prime}, G^{\prime \prime}, B$, if the following conditions are satisfied:

(1) To every triad $\mathfrak{F}^{\prime}, \mathfrak{F}^{\prime \prime}, \mathfrak{F}^{\prime} \times \mathfrak{F}^{\prime \prime}$ there exists a mapping $f: B \rightarrow A$ such that $\left(\mathbb{F}^{\prime} ; f: B\right) \equiv \mathfrak{F}^{\prime},\left(\mathbb{F}^{\prime \prime} ; f: B\right) \equiv \mathfrak{F}^{\prime \prime},\left(\mathbb{F}^{\prime} \times \mathbb{F}^{\prime \prime} ; f: B\right) \equiv \mathfrak{F}^{\prime} \times \mathfrak{F}^{\prime \prime}$.

(2) The three fibre bundles in the triads induced by the mappings $f_{0}, f_{1}: B \rightarrow A$ are respectively equivalent if and only if $f_{0} \simeq f_{1}$.

Relative to $F^{\prime}, F^{\prime \prime}, G^{\prime}, G^{\prime \prime}, B$ the problem on the existence of a universal triad of fibre bundles is solved by the following theorem: 
THEOREM 4.2. The triad $\left\{\mathbb{F}^{\prime}, \mathbb{F}^{\prime \prime} ; \mathbb{F}^{\prime} \times \mathbb{F}^{\prime \prime}\right\}$ is a universal triad of fibre bundles, relative to $F^{\prime}, F^{\prime \prime}, G^{\prime}, G^{\prime \prime}, B$, if and only if the fibre bundle $\mathbb{F}^{\prime} \times \mathbb{F}^{\prime \prime}$ is a universal fibre bundle relative to $F^{\prime} \times F^{\prime \prime}, G^{\prime} \times G^{\prime \prime}, B$.

The proof of this theorem is straightforward, and we shall omit it here.

5. The classical groups. We shall devote this section to exhibit explicitly the universal fibre bundles for the cases that the reference groups are the classical groups, namely, the general linear group $G L(n, R)$ with real coefficients, its subgroup $G L^{+}(n, R)$ of the linear transformations with positive determinant, the orthogonal group $O(n, R)$, the proper orthogonal group $S O(n, R)$, the unitary group $U(n, C)$, and the unitary symplectic group $S p(n)$.

1. The universal fibre bundles for the classical groups. The universal fibre bundles in question will be constructed according to the following general process: Let $R$ be a Lie group, and $K, H$ closed subgroups of $R, H \subset K$. Define the projection of $R / H$ onto $R / K$ as the mapping which maps the coset $r H$ into the coset $r K, r \in R$. With coordinate functions defined by the canonical coordinates, Steenrod $\left({ }^{11}\right)$ proved that a fibre bundle can be defined with the director space $K / H$, operated on by the group $K$ as left translations, and with the total space $R / H$ and the base space $R / K$. We shall denote such a fibre bundle by

$$
\{K / H, K ; R / H, R / K\} \text {, }
$$

omitting the projection and the coordinate functions, or simply by $\{R / H, R / K\}$, indicating only the total space and the base space.

To construct universal fibre bundles for the classical groups the general idea is to consider the same classical groups with more variables and construct their coset spaces. We begin with the following notations:

$G L(n)$ denotes the group of all $n$-rowed real matrices with nonzero determinant, $G L^{+}(n)$ the subgroup of $G L(n)$ consisting of all matrices of positive determinant. $H^{+}(n, N)$ and $H(n, N)$ denote respectively the subgroups of $G L^{+}(n, N)$ consisting of all matrices of the form

$$
\left(\begin{array}{cc}
G L^{+}(n) & 0 \\
* & G L^{+}(N)
\end{array}\right), \quad\left(\begin{array}{cc}
G L(n) & 0 \\
* & G L(N)
\end{array}\right),
$$

where the elements in the upper right corners are zero. $O(n)$ denotes the group of all $n$-rowed real orthogonal matrices and $S O(n)$ the subgroup of $O(n)$ consisting of all the matrices of determinant $+1 . U(n)$ denotes the group of all $n$-rowed unitary matrices, that is, matrices $\sigma$ with complex elements satisfying $\bar{\sigma}=\sigma^{\prime-1}$. For a quaternion $q=a_{0}+a_{1} i+a_{2} j+a_{3} k$ let $\bar{q}=a_{0}-a_{1} i-a_{2} j$ $-a_{3} k$ be its conjugate quaternion. Then $S p(n)$ denotes the group of all $n$ rowed matrices $\tau$ with quaternion elements satisfying $\bar{\tau}=\tau^{\prime-1}$.

(11) Steenrod [4, pp. 300-302]. 
When there are several groups in different numbers of variables, it is possible to imbed one in another. We agree by convention that the imbeddings $S O(N) \subset S O(n+N), S O(n) \times S O(N) \subset S O(n+N)$ are such that

$$
\left(\begin{array}{cc}
I_{n} & 0 \\
0 & S O(N)
\end{array}\right), \quad\left(\begin{array}{cc}
S O(n) & 0 \\
0 & S O(N)
\end{array}\right)
$$

and similarly for the other groups.

With all these definitions and conventions we consider the following fibre bundles:

$$
\begin{aligned}
\mathfrak{F}_{G L^{+}}(n, N) & =\left\{G L^{+}(n+N) / G L^{+}(N), G L^{+}(n+N) / H^{+}(n, N)\right\}, \\
\mathfrak{F}_{s o}(n, N) & =\{S O(n+N) / S O(N), S O(n+N) / S O(n) \times S O(N)\}, \\
\mathfrak{F}_{U}(n, N) & =\{U(n+N) / U(N), U(n+N) / U(n) \times U(N)\}, \\
\mathfrak{F}_{s p}(n, N) & =\{S p(n+N) / S p(N), S p(n+N) / S p(n) \times S p(N)\} .
\end{aligned}
$$

Take, for instance, $\mathfrak{F}_{s o}(n, N)$. Its director space is $S O(n) \times S O(N) / I_{n} \times S O(N)$, operated on by $S O(n) \times S O(N)$ as left translations. By a natural homeomorphism we can take $S O(n)$ for director space, which is then operated on by $S O(n)$ as left translations. It follows that $\mathfrak{F}_{s o}(n, N)$ is a principal fibre bundle. Similarly, we see that $\mathfrak{F}_{G L}+(n, N), \mathfrak{F}_{U}(n, N)$ and $\mathfrak{F}_{S p}(n, N)$ are principal fibre bundles. These fibre bundles will serve as universal principal fibre bundles for the cases that the reference groups are the classical groups, as given by the following theorem:

THEOREM 5.1. Let $B$ be a finite polyhedron of dimension $k$, and $\mathfrak{B}$ a universal principal fibre bundle relative to $G, G, B$. When $G$ is a classical group, a corresponding $\mathfrak{B}$ is given by the following table.

$G$

$$
\begin{aligned}
& S O(n) \\
& G L^{+}(n) \\
& U(n) \\
& S p(n)
\end{aligned}
$$

\section{$\mathfrak{B}$}

$$
\begin{aligned}
& \mathfrak{\mho}_{s o}(n, N) \\
& \mathfrak{\mho}_{G L^{+}}(n, N) \\
& \mathfrak{\mho}_{U}(n, N) \\
& \mathfrak{F}_{s_{p}}(n, N)
\end{aligned}
$$

Proof. It suffices to prove that the homotopy groups $\pi_{i}, 0 \leqq i \leqq k$, of the total spaces of $\mathfrak{B}$ are zero. As in the proof of Theorem 2.5, this follows from successive applications of the covering homotopy theorem. The desired deformations can be carried out, because the spaces $S O(N+1) / S O(N)$, $U(N+1) / U(N), S p(N+1) / S p(N)$ are homeomorphic to spheres of dimensions $N, 2 N+1,4 N+3$, respectively. This completes the proof of the theorem.

When the reference group is $G L(n)$ or $O(n)$, the situation differs slightly from the preceding ones, as these groups are not connected. In order that the foregoing manipulations prevail, we have to consider the fibre bundles 


$$
\begin{aligned}
\mathfrak{F}_{o}(n, N) & =\{S O(n+N) / S O(N), S O(n+N) / S O(n) \otimes S O(N)\}, \\
\mathfrak{F}_{G L}(n, N) & =\left\{G L^{+}(n+N) / G L^{+}(N), G L^{+}(n+N) / H(n, N)\right\},
\end{aligned}
$$

where

$$
S O(n) \otimes S O(N)=S O(n+N) \cap(O(n) \times O(N)) \text { in } O(n+N) .
$$

This makes the total spaces connected, and the proof of Theorem 5.1 can be applied. We state this result in the theorem:

THEOREM 5.2. Let $B$ be a finite polyhedron of dimension $k$. If $k \leqq N-1$, $\mathfrak{F}_{o}(n, N)$ and $\mathfrak{F}_{G L}(n, N)$ are universal principal fibre bundles relative to $O(n)$, $B$ and $G L(n), B$, respectively.

By the process $\tau_{F}^{-1}$ universal fibre bundles can be constructed whenever the reference group is one of the classical groups. We notice that the process $\tau_{F}^{-1}$ does not affect the base space. For the description of the characteristic ring it will be useful to know the base space, and in particular its cohomology ring.

We consider the linear vector spaces $V(n+N, R), V(n+N, C)$, $V(n+N, Q)$, of dimension $n+N$, over the real field, the complex field, and the quaternion field, respectively. Denote by $G(n, N, R), G(n, N, C), G(n, N, Q)$ respectively the manifolds of the linear spaces of dimension $n$ through the origin of these vector spaces. They are known as the Grassmann manifolds. For the case of the real field we may also consider the Grassmann manifold $\tilde{G}(n, N, R)$ of the oriented linear spaces of dimension $n$ through the origin. Each of these Grassmann manifolds is operated on transitively by the corresponding classical group in the vector space of dimension $n+N$, and we easily identify it with a space of cosets. In this way we deduce the theorem:

THEOREM 5.3. The base spaces of the universal fibre bundles for the classical groups are given by the following table.

$\begin{array}{cc}\text { Fibre Bundle } & \text { Base Space } \\ \mathfrak{F}_{G L^{+}}(n, N) & \tilde{G}(n, N, R) \\ \mathfrak{F}_{s_{0}}(n, N) & \tilde{G}(n, N, R) \\ \mathfrak{F}_{U}(n, N) & G(n, N, C) \\ \mathfrak{F}_{s_{p}}(n, N) & G(n, N, Q) \\ \mathfrak{F}_{o}(n, N) & G(n, N, R) \\ \mathfrak{F}_{G L}(n, N) & G(n, N, R)\end{array}$

2. Whitney's product for sphere bundles and a universal triad. From Theorem 5.3 we see that the universal sphere bundles and the universal vector bundles, whether oriented or non-oriented, have the same base space, 
namely, $\widetilde{G}(n, N, R)$ and $G(n, N, R)$, respectively. It follows from this and also from the considerations of $\$ 4.2$ that to each equivalence class of sphere bundles over a base space $B$ is associated an equivalence class of vector bundles, and vice versa. Denote these operations by $w$ and $w^{-1}$, respectively. Let $\mathfrak{F}_{1}$ and $\mathfrak{F}_{2}$ be two sphere bundles over the same base space $B$. The bundle $w^{-1}\left(w \mathfrak{F}_{1} \times w \mathfrak{F}_{2}\right)$ is then Whitney's product of the sphere bundles $\mathfrak{F}_{1}$ and $\mathfrak{F}_{2}$.

To construct a universal triad for the product $w_{1} \times w \mho_{2}$ suppose $m$ and $n$ be the dimensions of the director spaces of $w F_{1}$ and $w F_{2}$, respectively. Consider in $V(m+n+N, R)$ the manifold whose elements consist of a linear subspace of dimension $m$ and a linear subspace of dimension $n$ in general position through the origin. Call this manifold an E-manifold and denote it by $E(m, n, N, R)\left({ }^{12}\right)$. A mapping $B \rightarrow E(m, n, N, R)$ then induces two vector bundles and their product over $B$. From Theorem 4.1 it follows that $E(m, n, N, R)$ is the base space of a universal triad, provided that $\operatorname{dim} B \leqq N$ -1 . The study of the homology properties, and in particular of the cohomology ring, of $E(m, n, N, R)$ has therefore an important significance for the description of the position of one sphere bundle in another.

\section{BIBLIOGRAPHY}

1. C. Ehresmann, C. R. Acad. Sci. Paris vol. 213 (1941) pp. 762-764; vol. 214 (1942) pp. 144-147; vol 216 (1943) pp. 628-630.

2. S. T. Hu, Mappings of a normal space in an absolute neighborhood retract, Trans. Amer. Math. Soc. vol. 64 (1948) pp. 336-358.

3. W. Hurewicz, and N. Steenrod, Homotopy relations in fibre spaces, Proc. Nat. Acad. Sci. U.S.A. vol. 27 (1941) pp. 61-64. 294-311.

4. N. E. Steenrod, The classification of sphere bundles, Ann. of Math. vol. 45 (1944) pp.

5. H. Whitney, Topological properties of differentiable manifolds, Bull. Amer. Math. Soc. vol. 43 (1937) pp. 785-805.

6. - On the topology of differentiable manifolds, Lectures in Topology, University of Michigan Press 1941, pp. 101-141.

Academia Sinica, Nanking, China.

(12) The homology properties of the $E$-manifolds have been studied by Ehresmann; cf. C. Ehresmann, Journal de Mathématiques vol. 16 (1937) pp. 69-100. 